This document is confidential and is proprietary to the American Chemical Society and its authors. Do not copy or disclose without written permission. If you have received this item in error, notify the sender and delete all copies.

\title{
Photon Upconversion from Near-Infrared to Blue Light with TIPS-Anthracene as An Efficient Triplet-Triplet Annihilator
}

\begin{tabular}{|r|l|}
\hline Journal: & ACS Materials Letters \\
\hline Manuscript ID & tz-2019-002875.R2 \\
\hline Manuscript Type: & Letter \\
\hline Duthor: & 26-Oct-2019 \\
\hline Complete List of Authors: & $\begin{array}{l}\text { Nishimura, Naoyuki; University of Cambridge, Cavendish Laboratory; } \\
\text { Asahi Kasei Corporation Fuji Branch } \\
\text { Gray, Victor; University of Cambridge, Cavendish Laboratory; Uppsala } \\
\text { University, Department of Chemistry, Angström Laboratory } \\
\text { Allardice, Jesse; University of Cambridge, Physics } \\
\text { Zhang, Zhilong; University of Cambridge Department of Physics, } \\
\text { Cavendish Laboratory } \\
\text { Pershin, Anton; University of Mons, Laboratory for Chemistry of Novel } \\
\text { Materials } \\
\text { Beljonne, David; University of Mons, Laboratory for Chemistry of Novel } \\
\text { Materials } \\
\text { Rao, Akshay; University of Cambridge, Cavendish Laboratory }\end{array}$ \\
\hline
\end{tabular}

\section{SCHOLARONE Manuscripts}




\title{
Photon Upconversion from Near-Infrared to Blue Light with TIPS-Anthracene as An Efficient Triplet-Triplet Annihilator
}

\author{
Naoyuki Nishimura ${ }^{\dagger} \ddagger$, Victor Gray ${ }^{\dagger}, \S$, Jesse R. Allardice ${ }^{\dagger}$ Zhilong Zhang ${ }^{\dagger}$, Anton Pershin`${ }^{\wedge}$ \\ David Beljonne^, Akshay Rao*,† \\ † Cavendish Laboratory, University of Cambridge, J. J. Thomson Avenue, Cambridge, CB3 0HE, United Kingdom \\ ‡ Corporate Research \&Development, Asahi-Kasei Corporation, 2-1 Samejima, Fuji, Shizuoka, 416-8501, Japan \\ $\S$ Department of Chemistry, Ångström Laboratory, Uppsala University, Box 532, SE-751 20 Uppsala, Sweden \\ $\wedge^{\wedge}$ Laboratory for Chemistry of Novel Materials, University of Mons, Place du Parc 20, Mons, Belgium.
}

KEYWORDS: Photon upconversion, Triplet-triplet annihilation, 9,10-Bis[(triisopropylsilyl)ethynyl]anthracene,

\begin{abstract}
Photon upconversion (PUC) via triplet-triplet annihilation (TTA) from near-infrared (NIR) to blue photons could have important applications especially to bio-imaging and drug delivery accompanied by photochemical reaction. The fundamental challenges in achieving this has been the large anti-Stokes shift combined with the need to efficiently sensitize within the biological transparency window $(700-900 \mathrm{~nm})$. This calls for materials combinations with minimal energy losses during sensitization and minimal energy requirements to drive efficient TTA. Here, we demonstrate efficient PUC converting from NIR energy to blue photons using the commercially available material 9,10Bis[(triisopropylsilyl)ethynyl]anthracene (TIPS-Ac) as the annihilator. With a conventional triplet sensitizing system, TIPSAc performed TTA efficiency of $77 \pm 3 \%$ despite a relatively small driving force, compared to conventional TTA material converting from NIR to blue, for the TTA of less than $0.32 \mathrm{eV}$. Combined with Pt(II) meso-Tetraphenyl Tetrabenzoporphine (PtTPBP), which is a heavy atom triplet sensitizer that directly generates triplets upon NIR photon excitation, the resulting system allowed for an anti-Stokes shift of $1.03 \mathrm{eV}$. Our results highlight the use of direct triplet generation via NIR excitation as a useful path to achieving large anti-Stokes shift and also show that high TTA efficiencies can be achieved even in the absence of large driving energies for the TTA process.
\end{abstract}

Photon upconversion (PUC) via triplet-triplet annihilation (TTA) is an attractive means for solar energy concentration, bio-imaging, and photochemical reaction, such as photoinduced drug delivery. In particular,

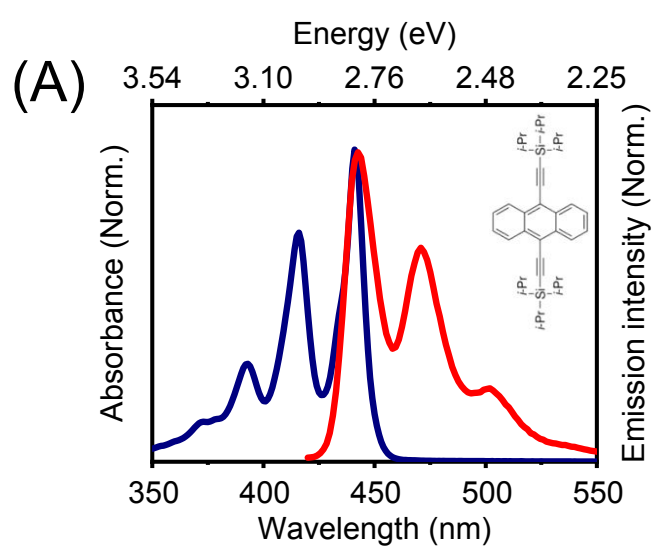

generation of blue light $(<500 \mathrm{~nm})$ via upconversion is important, because blue light is required for most photochemical reactions ${ }^{1,2}$, as well as photocatalytic water splitting to produce hydrogen, since most materials with

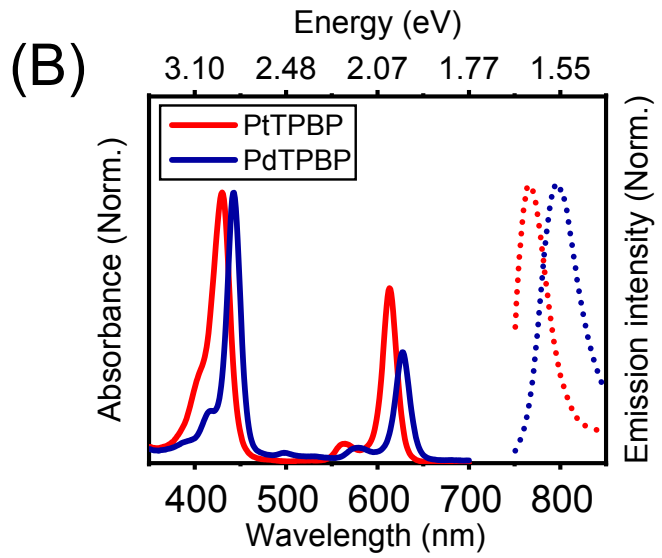

Figure 1. (A) absorption (blue) and emission (red) spectra of diluted TIPS-Ac in toluene, (B) absorption spectra (left) and emission spectra (right) of PdTPBP (blue) and PtTPBP(red) 
the efficient quantum yield for photocatalytic water splitting can harvest only photons with energy above 500 $\mathrm{nm} \quad$ (e.g., GaN:ZnO, $\mathrm{BiVO}_{4} \quad-\mathrm{SrTiO}_{3}: \mathrm{Rh}($,La $\left.)\right)^{3,4}$. For biomedical applications, the generation of blue photons is again important to trigger photoinduced drug delivery, photo-dynamic therapy and for optogenetics. In addition, for these applications it is crucial to utilizing near-infrared (NIR) photons to drive the upconversion, as the window for high transparency of biological tissue lies in the NIR region $(700-900 \mathrm{~nm}){ }^{1,5-8}$ While there are many systems with PUC working in the NIR to red,,$^{5,9-13}$ or green to blue regions, ${ }^{13-17}$ there are few systems capable to functioning to convert NIR to blue photons. ${ }^{6-8}$

As the difference in energy between blue and NIR photons is large (e.g., $470 \mathrm{~nm}: 2.64 \mathrm{eV}$ and $800 \mathrm{~nm}: 1.55$ $\mathrm{eV}$ ), a reduction of energy loss in PUC process, leading to larger anti-Stokes shift (: (PUC emission energy) (excitation energy)), is also important. ${ }^{6}$ However, achieving such a large anti-Stokes shift is extremely challenging and there have been very few reports of shifts greater than $1.0 \mathrm{eV} \cdot{ }^{6,8}$ Crucially, there has been no report, to the best of our knowledge, of a material with an antiStokes shift greater than $1.0 \mathrm{eV}$ that also has a linear excitation intensity dependency (slope 1 for the PUC process). The total energy loss in PUC processes arises from the following components: (1) energy loss from intersystem crossing (ISC) in the triplet sensitizer $\left(\mathrm{S}_{1}\right.$ sensitizer $-\mathrm{T}_{1}$ sensitizer $),(2)$ energy loss involved with triplet energy transfer (TET) from the sensitizer to the emitter, (3) energy loss due to the driving force for the TTA process ( $2 \mathrm{~T}_{1}$ annihilator $-\mathrm{S}_{1}$ annihilator). To overcome (1), the losses associated with generation of triplets via ISC, there have been reports of direct excitation from $S_{0}$ to $T_{1}$ in molecules containing heavy atoms (e.g., osmium (Os) complex, or acenes with bromide (Br) chromophore). However, such materials have not achieved an anti-Stokes shift of $1.0 \mathrm{eV}$ or above with a linear excitation intensity dependency. ${ }^{5-8}$

One of the keys to reduce loss in energy for PUC is to minimize the driving force of TTA process (i.e., process (3): difference in energy between $2 \mathrm{~T}_{1}$ and $\mathrm{S}_{1}$ of TTA material). Reports of annihilators fluorophores which can accept low-energy triplets $(<1.55 \mathrm{eV})$ and generate blue light have so far mainly focused on 9,10Bis(phenylethynyl)anthracene (BPEA) ${ }^{18-23}$ and perylene derivatives. ${ }^{1,7,24}$ However, these materials function with low efficiency of TTA (e.g., BPEA) $)^{18-20}$ and/or require large driving force for TTA (e.g., perylene derivative: $2 \mathrm{~T}_{1}-\mathrm{S}_{1} \approx$ $0.38 \mathrm{eV})^{1,7,24}$. Therefore, there is a clear need for PUC materials with a large anti-Stokes shift as well as efficient PUC from NIR to blue.

Herein, we show efficient TTA $\left(\Phi_{\mathrm{TTA}}=77 \pm 3 \%\right)$ in 9,10-Bis[(triisopropylsilyl)ethynyl]anthracene (TIPS-Ac) which can accept NIR energy and generate blue light (ca. $470 \mathrm{~nm}$ ). We demonstrate that the TTA process occurs efficiently, despite a relatively small driving force of less
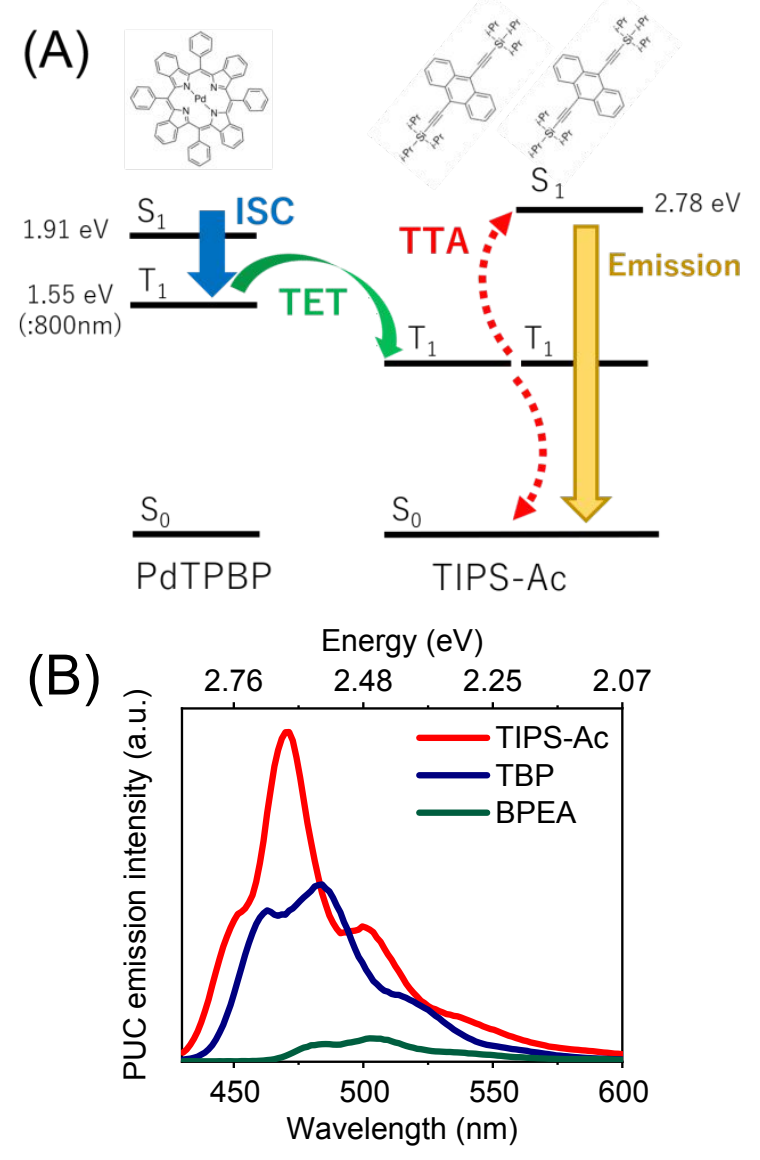

Figure 2. (A) Scheme of PUC with TIPS-Ac and PdTPBP via ISC triplet sensitization, (B) PUC emission spectra of solution containing $1 \mathrm{mM}$ TIPS-Ac, TBP, or BPEA and $20 \mathrm{uM}$ PdTPBP excited at $635 \mathrm{~nm}$ with power density of $6.0 \mathrm{~mW} \mathrm{~cm}^{-2}$

than $0.32 \mathrm{eV}$. This enables a large anti-Stokes shift of 1.03 $\mathrm{eV}$, when the triplet state of the sensitizer is directly excited with NIR light (at $785 \mathrm{~nm}$ ).

Figure $1 \mathrm{~A}$ shows absorption and emission spectra of diluted TIPS-Ac in toluene. TIPS-Ac possesses $S_{1}$ energy of $2.78 \mathrm{eV}$, which was estimated with extrapolation of the absorption spectrum, and emission with peaks at $430 \mathrm{~nm}$ $(0-0), \quad 470 \mathrm{~nm} \quad(0-1)$, and $500 \mathrm{~nm} \quad(0-2){ }^{25-29}$ The photoluminescence quantum yield (PLQY) of a $1 \mathrm{mM}$ TIPSAc toluene solution was measured to be $75 \pm 2 \%$, when excited at $405 \mathrm{~nm} .{ }^{30}$

We begin by investigating the TTA properties of TIPSAc, using a conventional sensitization process. Pd(II) meso-Tetraphenyl Tetrabenzoporphine (PdTPBP) is a porphyrin based triplet sensitizer which generate triplet excitons via ISC. ${ }^{31}$ The absorption and emission spectra of PdTPBP is depicted in Figure 1B. Figure 2A shows the PUC scheme. We compare TIPS-Ac with BPEA and 2,5,8,11-

Table 1. Parameters of PUC with TIPS-Ac, TBP, or BPEA and PdTPBP excited at $635 \mathrm{~nm}$

$\begin{array}{cccccc}\text { Sample } & \text { कUC (\%) } & \text { कISC (\%) } & \text { ФTET (\%) } & \text { कFL (\%) } & \text { ФTTA (\%) } \\ \text { TIPS-Ac + PdTPBP } & 27 \pm 0.5 & 97 & 97 & 75 \pm 2 & 77 \pm 3 \\ \text { TBP + PdTPBP } & 17.9 & 97 & 93 & 94 & 42 \\ \text { BPEA + PdTPBP } & 2.0 & 97 & 100 & 85 & 4.9\end{array}$


Tetra-tert-butylperylene (TBP: a perylene derivative with high solubility in non-polar solvents) using the same sensitizer and the concentration. Figure 2B depicts PUC spectra of solutions consisting of $1 \mathrm{mM}$ TIPS-Ac, BPEA, or TBP, and $20 \mathrm{uM}$ PdTPBP with an excitation at $635 \mathrm{~nm}$ of $6.0 \mathrm{~mW} \mathrm{~cm}^{-2}$. All samples generated PUC emission. However, under the same excitation conditions, the sample with TIPS-Ac generated the brightest emission of the three. The upconversion quantum yield (UCQY) for TIPS-Ac, TBP, BPEA are measured to be $27 \pm 0.5 \%, 17.9 \%, 2.0 \%$, respectively, against a maximum of $50 \%$ due to the twophoton nature of the process. We note that the UCQY was measured relative to BPEA in degassed toluene, which showed a PLQY of $85 \%{ }^{19}$. The obtained PUC PLQY of $2.0 \%$ in the BPEA system is similar to previous reports. ${ }^{18,19}$ Table 1 lists the parameters of the PUC calculated with the following equation (1).

$$
\Phi_{\mathrm{UC}}=1 / 2 \Phi_{\mathrm{ISC}} \times \Phi_{\mathrm{TET}} \times \Phi_{\mathrm{TTA}} \times \Phi_{\mathrm{FL}}
$$

The $\Phi_{\mathrm{UC}}, \Phi_{\mathrm{ISC}}, \Phi_{\mathrm{TET}}, \Phi_{\mathrm{TTA}}$, and $\Phi_{\mathrm{FL}}$ are represented as UCQY, ISC efficiency, TET efficiency, TTA efficiency, and PLQY of emission from the emitter (annihilator in this work), respectively. The $\Phi_{\text {ISC }}$ was taken to be 0.97 based on previous work. ${ }^{31} \Phi_{\text {TET }}$ was estimated via the quenching of phosphorescence at around $800 \mathrm{~nm}$ from PdTPBP in the presence of each annihilator. TET from PdTPBP $\left(\mathrm{T}_{1}\right.$ of 1.55 $\mathrm{eV}$ ) to TIPS-Ac was as efficient as $97 \%$, with negligible back-transfer in the quasi-steady state, suggesting that the triplet energy of TIPS-Ac lies below $1.55 \mathrm{eV}$. Also, since the TTA process occurs efficiently, the $2 \mathrm{~T}_{1}$ energy is estimated to be larger than the $S_{1}$ energy $(2.78 \mathrm{eV})$. We therefore estimate the triplet energy of TIPS-Ac to be in the region of 1.39 - $1.55 \mathrm{eV}$. This suggests a driving energy for the TTA process in the region of $0-0.32 \mathrm{eV}$. We note that direct phosphorescence measurements of the TIPS-Ac were inconclusive on the triplet energy.

The estimated TTA efficiency of TIPS-Ac (77 $\pm 3 \%)$ is highest in the series of the materials measured here, with TBP of $42 \%$, BPEA of $4.9 \%$. This is despite the relatively small driving force, compared to conventional TTA material converting from NIR to blue, for TTA in TIPS-Ac $\left(2 \mathrm{~T}_{1}-\mathrm{S}_{1}<0.32 \mathrm{eV}\right)$. This paves the way for the use of TIPSAc is an efficient TTA material which could achieve a large anti-Stokes shift and be capable of PUC from the NIR to blue. We note that all measurements in Figure $2 \mathrm{~B}$ and Table 1 were carried out in quasi-linear regime (slope of ca. 1.2), where TTA efficiency is maximized. The PUC in emission intensity vs excitation power density data are shown in Figure S1. We also note that while the numbers of $\Phi_{\mathrm{FL}}$ in TBP and BPEA in Table 1 were taken from literatures ${ }^{7,19}$, our absolute measurement for fluorescence resulted in similar numbers to the literatures (TBP: $89 \%$, BPEA: $82 \%$ ), and hence, what we are proving of high efficiency in TTA with TIPS-Ac here is evidenced.

In order to demonstrate the utilization of NIR photons for PUC with TIPS-Ac annihilator, a triplet sensitizer with heavier atom was used in the PUC system. Pt(II) mesoTetraphenyl Tetrabenzoporphine (PtTPBP) is known for generating triplet exciton via ISC, with a $\mathrm{T}_{1}$ energy of around $1.61 \mathrm{eV}$ judged from energy of the phosphorescence at room temperature ${ }^{32}$ (Figure 1B).
Here, we have found another route to generate triplet in PtTPBP. The absorption spectrum of PtTPBP solution in NIR region (Figure S2A) has a broad absorption with a peak at around $755 \mathrm{~nm}(1.64 \pm 0.6 \mathrm{eV})$, with linear dependence of optical density on the concentration (Figure S2B). This is assigned as the $\mathrm{S}_{0}-\mathrm{T}_{1}$ transition in PtTPBP, allowing us to directly excite the triplets with NIR photons, followed by the PUC, as shown in Figure 3A.
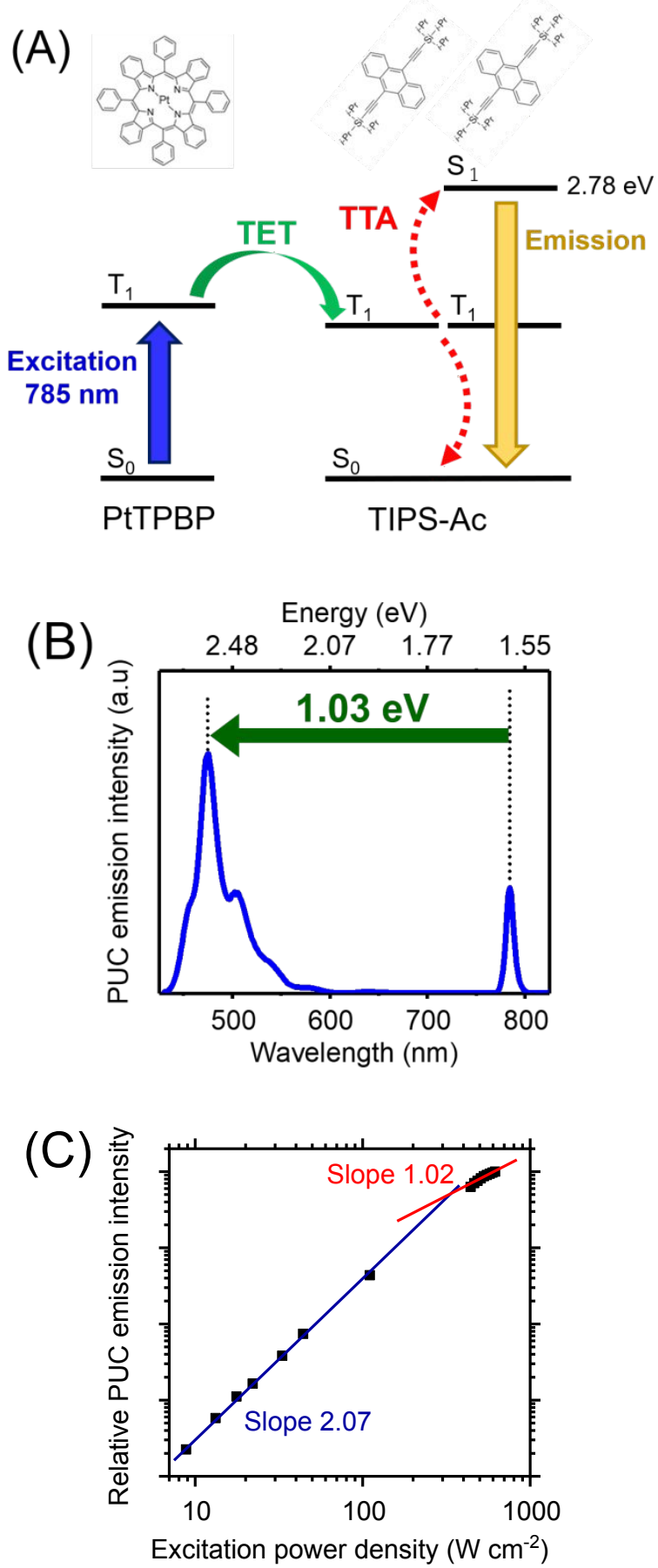

Figure 3. (A) Scheme of PUC with TIPS-Ac and PtTPBP via direct triplet sensitization, (B) PUC emission spectra of solution containing $1 \mathrm{mM}$ TIPS-Ac and $1 \mathrm{mM}$ PtTPBP excited at $785 \mathrm{~nm},(\mathrm{C})$ relative PUC emission intensity against excitation power dependency of $1 \mathrm{mM}$ TIPS-Ac and $1 \mathrm{mM}$ PtTPBP excited at $785 \mathrm{~nm}$ 
Figure $3 \mathrm{~B}$ shows the PUC emission spectrum of a solution consisting of $1 \mathrm{mM}$ TIPS-Ac and $1 \mathrm{mM}$ PtTPBP, filled in a $1 \mathrm{~mm}$ path length cuvette, excited at $785 \mathrm{~nm}$. In the PUC emission spectrum, the peak at around $785 \mathrm{~nm}$ was derived from the scattering of the excitation laser. PUC emission with a peak at $475 \mathrm{~nm}$ arising from TIPS-Ac was observed. This constitutes an anti-Stokes shift of $1.03 \mathrm{eV}$. The apparent slight red shift from the original (0-1) emission at $470 \mathrm{~nm}$ to the observed $475 \mathrm{~nm}$ was likely due to reabsorption by TIPS-Ac and/or PtTPBP. Although the (0-0) emission (at $430 \mathrm{~nm}$ ) of TIPS-Ac was almost completely suppressed due to reabsorption, the antiStokes shift achieved is over $1.0 \mathrm{eV}$. This is made possible by the relatively small driving force for TTA $\left(2 \mathrm{~T}_{1}-\mathrm{S}_{1}<0.32\right.$ $\mathrm{eV}$ ) compared to conventional TTA material such as TBP (2 $\mathrm{T}_{1}-\mathrm{S}_{1} \approx 0.38 \mathrm{eV}$ ). Figure $3 \mathrm{C}$ shows the excitation power dependence of the TIPS-Ac - PtTPBP system. In the dependence, a change in the slope from square (2.07) to near linear (1.02) was observed, which is an evidence for TTA PUC. This is, to the best of our knowledge, the first report to achieve anti-Stokes shift of over $1.0 \mathrm{eV}$ with a linear slope in excitation power dependence. Also importantly, the $785 \mathrm{~nm}$ excitation used lies directly in the biological transparency window (700 - $900 \mathrm{~nm}$ ), allowing for long penetration depths.

We note that no contribution of two photon absorption was observed, as judged by measurement with $1 \mathrm{mM}$ TIPSAc solution, as a reference sample, excited at $785 \mathrm{~nm}$ with

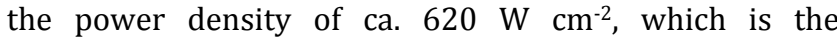
maximum in Figure 3C. The estimated PUC PLQY of a solution consisting of $1 \mathrm{mM}$ TIPS-Ac and $1 \mathrm{mM}$ PtTPBP system with an excitation at $785 \mathrm{~nm}$ (i.e., via direct triplet excitation) was $2.1 \%$. As a control measurement, the same solution (i.e., $1 \mathrm{mM}$ TIPS-Ac and $1 \mathrm{mM}$ PtTPBP) was excited at $635 \mathrm{~nm}$ (i.e., triplet generation via ISC) and resulted in a UCPY of $4.4 \%$ (this excitation power dependency: Figure S3).

At a high concentration of the triplet sensitizer, quenching of annihilator would occur (i.e., TTA material), resulting in a decrease in UCQY.19,33 While excitation conditions are different between continuous wave laser and pulse laser, time resolved emission measurement (supporting S4) indicates rate constant of quenching for the annihilator (TIPS-Ac) by the sensitizer (PtTPBP) was estimated to be $k_{\mathrm{q}}=8.0 \pm 0.4 \times 10^{7} \mathrm{M}^{-1} \mathrm{~s}^{-1}$. Therefore, most of the drop over $75 \%$ in the UCQY from the sample with $20 \mathrm{uM}$ PdTPBP $(27 \pm 0.5 \%)$ to the sample with $1 \mathrm{mM}$ PtTPBP (4.4\%) was due to quenching of annihilator by high concentration of the sensitizer. Self-annihilation of PtTPBP with the high concentration may also reduce the TET efficiency as the high concentration resulted in shorter lifetime of the phosphorescence (supporting S4). Besides, part of the decrease in the UCQY could also be attributed to reabsorption by the concentrated PtTPBP. On the other hand, the difference in the PLQY with $1 \mathrm{mM}$ PtTPBP between direct triplet excitation (2.1\%) and via ISC $(4.4 \%)$ was mainly due to reabsorption. For application to bio-imaging and/or drug delivery, the upconverter material would be packed in space of $100 \mu \mathrm{m}$ order size, for example in capsule delivered to a specific region of the body. ${ }^{22,23}$ For such applications, thus, reabsorption would be reduced and the $1 \mathrm{mM}$ TIPS-Ac and
$1 \mathrm{mM}$ PtTPBP would show PUC PLQY with NIR excitation as low as $4.4 \%$ or higher than that since even at $635 \mathrm{~nm}$ the experimental optical density of the sample was ca. 0.4, which is still capable of inducing significant reabsorption.

A prominent feature of this work is that we show TIPSAc to be a highly efficient TTA material $\left(\Phi_{\mathrm{TTA}}=77 \pm 3 \%\right)$, which is higher than BPEA (4.9\%) and diphenyl anthracene (DPA, $\sim 50 \%)^{19}$, despite their similar molecule structures. While there is an active debate in the literature as to the cause of these varying efficiencies it is worth noting the suggestion that a possible loss pathway for BPEA could be access to the $\mathrm{T}_{2}$ state via TTA. ${ }^{34}$ There is also the possibility of tuneable energies in the excited states due to the rotation of the ethynyl - phenyl chromophore, which could cause multi-molecule interactions involved in intermediate states in TTA process to relax in an unfavorable manner. ${ }^{19}$ To understand the energetic structure of TIPS-Ac we have performed electronic structure calculations, to obtain the energy levels for this system. We find that TIPS-Ac has larger $\mathrm{T}_{2}$ energy than twice of $\mathrm{T}_{1}$ energy $\left(\mathrm{T}_{2}-2 \mathrm{~T}_{1}=0.06-0.83 \mathrm{eV}\right.$, Table S3), which is very important to reduce losses in the TTA process, as the $\mathrm{T}_{2}$ state is not accessible. Compared to DPA, TIPS-Ac possesses a more rigid bonding in ethynyl group and more symmetric structure, which may reduce non-radiative loss pass in the TTA process. Besides, the (triisopropylsilyl)ethynyl group would lead to preferable bi-molecule separation to proceeding TTA. These features may contribute to the higher TTA efficiency than DPA.

In conclusion, we have demonstrated PUC from NIR to blue photons with TIPS-Ac as an annihilator. Exciting PdTPBP at $635 \mathrm{~nm}$, generating triplets via ISC, showed a PUC PLQY with TIPS-Ac of $27 \pm 0.5 \%$ with a TTA efficiency of $77 \pm 3 \%$, despite a relatively small driving energy for the TTA of less than $0.32 \mathrm{eV}$. Utilizing direct triplet generation in PtTPBP via $785 \mathrm{~nm}$ excitation allowed for PUC with an anti-Stokes shift of $1.03 \mathrm{eV}$ to be achieved. The PLQY was reduced due to reabsorption losses with the measurements here, but for applications using sub-micro size, such as bio-imaging and drug delivery, these reabsorption effects would be reduced. As another strategy to overcome reabsorption losses, TIPS-Ac itself can function as photocatalyst ${ }^{26}$. These results suggest that direct excitation of triplets with NIR photons, combined with novel TTA emitters with low driving energies for TTA, yet capable of high PUC efficiency, offers a promising route to efficient NIR to blue upconversion.

\section{ASSOCIATED CONTENT}

The supporting information is available free of charge via the Internet at http://pubs.acs.org.

Experimental methods, excitation power dependence on PUC emission from TIPS-Ac, TBP, or BPEA and PdTPBP system, absorption spectrum of PtTPBP

\section{AUTHOR INFORMATION}

\section{Corresponding Author \\ * Email: ar525@cam.ac.uk \\ Notes}

The authors declare no competing financial interest 


\section{ACKNOWLEDGMENT}

The authors thank the Winton Programme for the Physics of Sustainability and the Engineering and Physical Sciences Research Council for funding. V. G. acknowledges funding from the Swedish Research Council, Vetenskapsrådet 201800238. AP acknowledges the financial support from the Marie Curie Fellowship (MILORD project, $\mathrm{N}^{\circ} .748042$ ). BD is an FNRS Research Director.

\section{REFERENCES}

1. Ravetz, B. D.; Pun, A. B.; Churchill, E. M.; Congreve, D. N.; Rovis, T.; Campos, L. M. Photoredox catalysis using infrared light via triplet fusion upconversion. Nature 2019, 565, 343346.

2. Smith, A. M.; Mancini, M. C.; Nie, S. Bioimaging: Second window for in vivo imaging. Nat. Nanotechnol. 2009, 4, 710711.

3. Maeda, K.; Teramura, K.; Lu, D.; Takata, T.; Inoue, Y.; Domen, K. Photocatalyst releasing hydrogen from water, Nature 2006, 440, 295.

4. Wang, Q.; Hisatomi, T.; Jia, Q.; Tokudome, H.; Zhong, M.; Wang, C.; Pan, Z.; Takata, T.; Nakabayashi, M.; Shibata, N.; Li, Y.; Sharp, I. D.; Kudo, A.; Yamada, T.; Domen, K. Scalable water splitting on particulate photocatalyst sheets with a solar-to-hydrogen energy conversion efficiency exceeding 1\%. Nat. Mater. 2016, 15, 611-615.

5. Amemori, S.; Sasaki, Y.; Yanai, N.; Kimizuka, N. Near-Infraredto-Visible Photon Upconversion Sensitized by a Metal Complex with Spin-Forbidden yet Strong S0-T1Absorption. J. Am. Chem. Soc. 2016, 138, 8702-8705.

6. Yanai, N.; Kimizuka, N. New Triplet Sensitization Routes for Photon Upconversion: Thermally Activated Delayed Fluorescence Molecules, Inorganic Nanocrystals, and Singletto-Triplet Absorption. Acc. Chem. Res. 2017, 50, 2487-2495.

7. Sasaki, Y.; Amemori, S.; Kouno, H.; Yanai, N.; Kimizuka, N. Near infrared-to-blue photon upconversion by exploiting direct S-T absorption of a molecular sensitizer. J. Mater. Chem. C 2017, 5, 5063-5067.

8. Okumura, K.; Matsuki, M.; Yamada, T.; Yanai, N.; Kimizuka, N. Sensitizer-Free Photon Upconversion in Single-Component Brominated Aromatic Crystals. ChemistrySelect 2017, 2, 7597-7601.

9. Singh-Rachford, T. N.; Castellano, F. N. Pd(II) phthalocyaninesensitized triplet - Triplet annihilation from rubrene. J. Phys. Chem. A 2008, 112, 3550-3556.

10. Amemori, S.; Yanai, N.; Kimizuka, N. Metallonaphthalocyanines as triplet sensitizers for nearinfrared photon upconversion beyond $850 \mathrm{~nm}$. Phys. Chem. Chem. Phys. 2015, 17, 22557-22560.

11. Wu, M.; Congreve, D. N.; Wilson, M. W. B.; Jean, J.; Geva, N.; Welborn, M.; Van Voorhis, T.; Bulović, V.; Bawendi, M. G.; Baldo, M. A. Solid-state infrared-to-visible upconversion sensitized by colloidal nanocrystals. Nat. Photonics 2015, 10, 31-34.

12. Nishimura, N.; Allardice, J. R.; Xiao, J.; Gu, Q.; Gray, V.; Rao, A. Photon upconversion utilizing energy beyond the band gap of crystalline silicon with a hybrid TES-ADT/PbS quantum dots system. Chem. Sci. 2019, 10, 4750-4760.

13. Huang, Z.; Li, X.; Mahboub, M.; Hanson, K. M.; Nichols, V. M.; Le, H.; Tang, M. L.; Bardeen, C. J. Hybrid MoleculeNanocrystal Photon Upconversion Across the Visible and Near-Infrared. Nano Lett. 2015, 15, 5552-5557.

14. Keivanidis, P. E.; Baluschev, S.; Miteva, T.; Nelles, G.; Scherf, U.; Yasuda, A.; Wegner, G. Up-Conversion Photoluminescence in Polyfluorene Doped with Metal(II)-Octaethyl Porphyrins. Adv. Mater. 2003, 15, 2095-2098.
15. Islangulov, R. R.; Kozlov, D. V.; Castellano, F. N. Low power upconversion using MLCT sensitizers. Chem. Commun. 2005 1, 3776-3778.

16. Simon, Y. C.; Weder, C. Low-power photon upconversion through triplet-triplet annihilation in polymers. J. Mater. Chem. 2012, 22, 20817-20830.

17. Haruki, R.; Kouno, H.; Hosoyamada, M.; Ogawa, T.; Yanai, N.; Kimizuka, N. Oligo(ethylene glycol)/alkyl-modified Chromophore Assemblies for Photon Upconversion in Water. Chem. Asian J. 2019, 14, 1723-1728.

18. Baluschev, S.; Yakutkin, V.; Miteva, T.; Wegner, G.; Roberts, T.; Nelles, G.; Yasuda, A.; Cheprakov, A. A general approach for non-coherently excited annihilation up-conversion: Transforming the solar-spectrum. New J. Phys. 2008, 10, 013007

19. Gray, V.; Dreos, A.; Erhart, P.; Albinsson, B.; Moth-Poulsen, K.; Abrahamsson, M. Loss channels in triplet-triplet annihilation photon upconversion: Importance of annihilator singlet and triplet surface shapes. Phys. Chem. Chem. Phys. 2017, 19, 10931-10939.

20. Singh-Rachford, T. N.; Castellano, F. N. Supra-nanosecond dynamics of a red-to-blue photon upconversion system. Inor Chem. 2009, 48, 2541-2548.

21. Moor, K.; Kim, J. H.; Snow, S.; Kim, J. H. [C70] Fullerenesensitized triplet-triplet annihilation upconversion. Chem. Commun. 2013, 49, 10829-10831.

22. Kang, J. H.; Reichmanis, E. Low-threshold photon upconversion capsules obtained by photoinduced interfacial polymerization. Angew. Chem. - Int. Ed. 2012, 51, 1184111844.

23. Kwon, O. S.; Song, H. S.; Conde, J.; Kim, H. Il; Artzi, N.; Kim, J. H. Dual-color emissive upconversion nanocapsules for differential cancer bioimaging in vivo. ACS Nano 2016, 10, 1512-1521.

24. Hoseinkhani, S.; Tubino, R.; Meinardi, F.; Monguzzi, A. Achieving the photon up-conversion thermodynamic yield upper limit by sensitized triplet-triplet annihilation. Phys. Chem. Chem. Phys. 2015, 17, 4020-4024.

25. Griffith, O. L.; Jones, A. G.; Anthony, J. E.; Lichtenberger, D. L. Intermolecular effects on the hole states of triisopropylsilylethynyl- substituted oligoacenes. J. Phys. Chem. C 2010, 114, 13838-13845.

26. Tehfe, M. A.; Lalevée, J.; Morlet-Savary, F.; Graff, B.; Blanchard, N.; Fouassier, J. P. Organic photocatalyst for polymerization reactions: 9,10- Bis[(triisopropylsilyl)ethynyl]anthracene. ACS Macro Lett. 2012, 1, 198-203.

27. Jun, Y. Time-Dependent Density Functional Theory Study of Low-Lying Absorption and Fluorescence Band Shapes for Phenylene-Containing Oligoacenes. J. Phys. Chem. A 2015, 119, 12706-12714.

28. Bhattacharyya, K.; Datta, A. Polymorphism controlled singlet fission in tips-Anthracene: Role of stacking orientation. $J$. Phys. Chem. C 2017, 121, 1412-1420.

29. Pun, J. K. H.; Gallaher, J. K.; Frazer, L.; Prasad, S. K. K.; Dover, C. B.; MacQueen, R. W.; Schmidt, T. W. TIPS-anthracene: a singlet fission or triplet fusion material? J. Photonics Energy 2018, 8,1 .

30. De Mello, J. C.; Wittmann, H. F.; Friend, R. H. An improved experimental determination of external photoluminescence quantum efficiency. Adv. Mater. 1997, 9, 230-232.

31. Rogers, J. E., Nguyen, K. A., Hufnagle, D. C., McLean, D. G., Su, W., Gossett, K. M.; Burke A. R.; Vinogradov, S. A.; Pachter R.; Fleitz, P. A. Observation and Interpretation of Annulated Porphyrins: Studies on the Photophysical Properties of meso-Tetraphenylmetalloporphyrins. J. Phys. Chem. A 2003, 107, 11331-11339.

32. Borek, C.; Hanson, K.; Djurovich, P. I.; Thompson, M. E.; Aznavour, K.; Bau, R.; Sun, Y.; Forrest, S. R.; Brooks, J.; Michalski, L.; Brown, J. Highly efficient, near-infrared 
electrophosphorescence from a Pt-metalloporphyrin complex. Angew. Chemie - Int. Ed. 2007, 46, 1109-

33. Gholizadeh, E. M.; Frazer, L.; MacQueen, R. W.; Gallaher, J. K.; Schmidt, T. W. Photochemical upconversion is suppressed by high concentrations of molecular sensitizers. Phys. Chem. Chem. Phys. 2018, 20, 19500-19506.

34. Schmidt, T. W.; Castellano, F. N. Photochemical upconversion: The primacy of kinetics. J. Phys. Chem. Lett. 2014, 5, 4062-4072. 